\title{
Static Output Feedback Control for Discrete-Time Switched Systems via Improved Path-Following Method
}

\author{
Jian Chen ${ }^{1,2}$ and Chong Lin ${ }^{2}$ \\ ${ }^{1}$ College of Science, Qingdao Technological University, Qingdao 266555, China \\ ${ }^{2}$ Institute of Complexity Science, Qingdao University, Qingdao 266071, China \\ Correspondence should be addressed to Jian Chen; janec@163.com
}

Received 12 May 2015; Accepted 15 June 2015

Academic Editor: Juan R. Torregrosa

Copyright (c) 2015 J. Chen and C. Lin. This is an open access article distributed under the Creative Commons Attribution License, which permits unrestricted use, distribution, and reproduction in any medium, provided the original work is properly cited.

\begin{abstract}
This paper focuses on the problems of static output feedback control and $H_{\infty}$ controller design for discrete-time switched systems. Based on piecewise quadratic Lyapunov functions and a new linearization method, new sufficient conditions for system stability and $H_{\infty}$ controller design are obtained. Then, an improved path-following algorithm is built to solve the problems. Finally, the merits and effectiveness of the proposed method are shown by two numerical examples.
\end{abstract}

\section{Introduction}

Recently, there has been an increasing interest in the study of switched systems because a wide class of nonlinear systems are naturally written as switched systems [1]. Moreover, many other types of nonlinear systems can also be modeled as switched systems approximately [2]. Switched systems are a particular class of hybrid systems which are bounded together by a switching rule. Such systems can be used to describe a wide range of physical and engineering systems in practice [3].

There are a large number of literatures about the stability analysis and design of switched systems during the last few years [4-9]. For discrete-time systems, several sufficient conditions have been presented based on different Lyapunov functions $[6,7,10,11]$, which are different in the conservative level and in the numerical difficulties. Previous work has concentrated on the output feedback controller design methods for switched systems based on piecewise quadratic Lyapunov function [12, 13]. Lyapunov-based controller synthesis is formulated as a biconvex optimization problem which is nonconvex, NP-hard, and very expensive to solve globally [14]. Although there exist some results in solving this problem that the corresponding conditions can be determined by checking a set of linear matrix inequalities (LMIs) [12, 13], most of them are very restrictive.
Path-following method, which is an effective method for solving the biconvex optimization problem, was proposed by Hassibi et al. [15] and employed to solve mixed $\mathrm{H}_{2} / \mathrm{H}_{\mathrm{\infty}}$ control $[16,17]$ and other control problems [18]. An improved path-following method [19] has enhanced the convergence and the performance of the algorithm. As a step-by-step method, implying linearization approach at its key step, it gradually shows enormous potential in solving control problems.

In this paper, the problem of static output feedback (SOF) control for discrete-time switched systems is studied. Based on piecewise quadratic Lyapunov functions $[12,13]$ and a new linearization method [19], the piecewise quadratic stability conditions are linearized around some points. As a result, less conservative conditions for system stability are derived. The problems of $H_{\infty}$ control design can be readily treated as well. Then, based on an improved path-following method, an iterative algorithm is built. Finally, two examples are given to show the merits and effectiveness of our work.

This paper is organized as follows. Section 2 is the problem formulation and preliminaries. Section 3 gives the SOF controller design for switched systems. Section 4 extends the method to $H_{\infty}$ SOF control. Section 5 provides two numerical examples to show the merits and effectiveness of the results and Section 6 concludes this paper. 
Notation. $R^{n}$ denotes the $n$-dimensional Euclidean space; the superscripts -1 and $T$ denote the matrix inverse and transpose, respectively; $X>0(X \geq 0)$ means that $X$ is positive definite (positive semi-definite); $\|\cdot\|$ is the spectral norm; the star $*$ denotes the symmetric term in a matrix; $l(X, Y)=X Y+X^{T} Y^{T}$.

\section{Problem Formulation and Preliminaries}

Consider the discrete-time switched system:

$$
\begin{aligned}
x(k+1) & =A_{i} x(k)+B_{i} u(k), \\
y(k) & =C_{i} x(k),
\end{aligned}
$$

for $x \in S_{i}, i=1,2, \ldots, l$,

where $x(k) \in R^{n}, u(k) \in R^{m}$, and $y(k) \in R^{p}$ are the state, the control input, and the measured output, respectively; $S_{i} \subseteq R^{n}$, $i=1,2, \ldots, l$, denotes a partition of the output space into a number of closed polyhedral regions. For future use, define a set $\Omega$ to represent all possible switches from one region to itself or another region; that is

$$
\begin{aligned}
\Omega & =\left\{(i, j): i, j=1,2, \ldots, l, \text { s.t. } x(k) \in S_{i}, x(k+1)\right. \\
& \left.\in S_{j}\right\} .
\end{aligned}
$$

The set $\Omega$ can be determined by the reachability analysis for mixed logical dynamical systems. The system is allowed to switch arbitrarily between subsystems.

We study the problem of designing a static output feedback controller:

$$
u(k)=F_{i} y(k), \quad i=1,2, \ldots, l,
$$

$$
\left(\begin{array}{ccc}
-\left(P_{i}(k)+\Delta P_{i}\right) & * & * \\
\left(P_{j}(k)+\Delta P_{j}\right) A_{c i}(k)+P_{j}(k) B_{i} \Delta F_{i} C_{i} & -\left(P_{j}(k)+\Delta P_{j}\right) & * \\
B_{i} \Delta F_{i} C_{i} & \Delta P_{j} & -2 I
\end{array}\right)<0,
$$

is stable.

The following lemmas give the stability condition of closed-loop systems (4) and the new linearization method proposed in [19].

Lemma 1 (see [12]). If there exist matrices $P_{i}=P_{i}^{T}>0(\forall i=$ $1,2, \ldots, l)$, such that the positive definite function $V(x)=$ $x^{T} P_{i} x\left(\forall x \in S_{i}\right)$, satisfies $V(x(k+1))-V(x(k))<0$, that is,

$$
\left(A_{i}+B_{i} F_{i} C_{i}\right)^{T} P_{j}\left(A_{i}+B_{i} F_{i} C_{i}\right)-P_{i}<0, \quad \forall i, j \in \Omega,
$$

then the closed-loop switched systems (4) are exponentially stable.

Lemma 2 (see [19]). If there exists a fixed point $(M, N)$ such that the LMI

$$
\left(\begin{array}{cc}
l(M+\Delta A, N)+l(M, \Delta P) & * \\
\Delta A^{T}+\Delta P & -2 I
\end{array}\right)<0
$$

holds for some $(\triangle A, \Delta P)$, then the point $(A=M+\Delta A, P=$ $N+\Delta P)$ is a feasible solution to the bilinear matrix inequality $(B M I)$

$$
l(A, P)<0 .
$$

\section{SOF Controller Design}

In this section, based on a piecewise quadratic Lyapunov function and the new linearization method, we will give new sufficient conditions for solving this problem.

Theorem 3. If there exist points $\left(F_{i}(k), P_{i}(k)\right), i=1,2, \ldots, l$, such that the following inequalities:

$$
P_{i}(k)+\Delta P_{i}>0, \forall(i, j) \in \Omega,
$$

hold for some $\left(\Delta F_{i}, \Delta P_{i}\right)$, where $A_{c i}(k)=A_{i}+B_{i} F_{i}(k) C_{i}$, then the points $\left(P_{i}=P_{i}(k)+\Delta P_{i}, F_{i}=F_{i}(k)+\Delta F_{i}\right)$ are feasible solutions to inequalities (5).

Proof. By Schur complement, (5) are equivalent to

$$
\left(\begin{array}{cc}
-P_{i} & \left(A_{i}+B_{i} F_{i} C_{i}\right)^{T} P_{j} \\
P_{j}\left(A_{i}+B_{i} F_{i} C_{i}\right) & -P_{j}
\end{array}\right)<0 .
$$

Write $F_{i}=F_{i}(k)+\Delta F_{i}, P_{i}=P_{i}(k)+\Delta P_{i}$, and $A_{c i}(k)=A_{i}+$ $B_{i} F_{i}(k) C_{i}$, where $F_{i}(k)$ and $P_{i}(k)$ are fixed matrices. The left side of inequality $(9)$ is expanded around $\left(F_{i}(k), P_{i}(k)\right)$ as

$$
\begin{aligned}
& \left(\begin{array}{cc}
-P_{i} & \left(A_{i}+B_{i} F_{i} C_{i}\right)^{T} P_{j} \\
P_{j}\left(A_{i}+B_{i} F_{i} C_{i}\right) & -P_{j}
\end{array}\right) \\
& =\left(\begin{array}{c}
-\left(P_{i}(k)+\Delta P_{i}\right) \\
\left(P_{j}(k)+\Delta P_{j}\right)\left(A_{i}+B_{i}\left(F_{i}(k)+\Delta F_{i}\right) C_{i}\right)-\left(P_{j}(k)+\Delta P_{j}\right)
\end{array}\right)
\end{aligned}
$$




$$
\begin{aligned}
& =\left(\begin{array}{cc}
-\left(P_{i}(k)+\Delta P_{i}\right) & * \\
\left(P_{j}(k)+\Delta P_{j}\right) A_{c i}(k)+P_{j}(k) B_{i} \Delta F_{i} C_{i} & -\left(P_{j}(k)+\Delta P_{j}\right)
\end{array}\right) \\
& +l\left(\left(B_{i} \Delta F_{i} C_{i} 0\right)^{T},\left(\begin{array}{ll}
0 & \Delta P_{j}
\end{array}\right)\right)<0 ;
\end{aligned}
$$

that is

$$
\begin{aligned}
& l\left(\left(B_{i} \Delta F_{i} C_{i} \quad 0\right)^{T},\left(\begin{array}{ll}
0 & \Delta P_{j}
\end{array}\right)\right) \\
& <\left(\begin{array}{cc}
P_{i}(k)+\Delta P_{i} & * \\
-\left(P_{j}(k)+\Delta P_{j}\right) A_{c i}(k)-P_{j}(k) B_{i} \Delta F_{i} C_{i} & P_{j}(k)+\Delta P_{j}
\end{array}\right) .
\end{aligned}
$$

Thus, by Lemma 2, inequalities (8) hold.
With Theorem 3, the nonlinear SOF optimization problem OP1

$$
\begin{aligned}
\min & \alpha \\
\text { subject to } & P_{i}>0 \\
& \left(\begin{array}{cc}
-P_{i}-\alpha P_{i} & \left(A_{i}+B_{i} F_{i} C_{i}\right)^{T} P_{j} \\
P_{j}\left(A_{i}+B_{i} F_{i} C_{i}\right) & -P_{j}
\end{array}\right)<0
\end{aligned}
$$

can be replaced by solving the following linear optimization problem OP1':

$$
\begin{aligned}
\min & \alpha \\
\text { subject to } & P_{i}(k)+\Delta P_{i}>0 \\
& \left(\begin{array}{cc}
\beta P_{i}(k) & \Delta P_{i} \\
\Delta P_{i} & \beta P_{i}(k)
\end{array}\right)>0 \\
& \left(\begin{array}{ccc}
-\left(P_{i}(k)+\Delta P_{i}\right)-\alpha P_{i}(k) & * & * \\
\left(P_{j}(k)+\Delta P_{j}\right) A_{c i}(k)+P_{j}(k) B_{i} \Delta F_{i} C_{i} & -\left(P_{j}(k)+\Delta P_{j}\right) & * \\
B_{i} \Delta F_{i} C_{i} & \Delta P_{j} & -2 I
\end{array}\right)<0 .
\end{aligned}
$$

Remark 4. In $\mathrm{OP} 1^{\prime}$, additional inequalities $\left(\begin{array}{ccc}\beta P_{i}(k) & \Delta P_{i} \\ \Delta P_{i} & \beta P_{i}(k)\end{array}\right)>0$ are added. This inequalities are the variation of inequalities $\left\|\Delta P_{i}\right\|<\beta\left\|P_{i}(k)\right\|$. Due to our new linearization method, the prescribed scalar $\beta$ should be small [19]. Otherwise, the conservation will increase.

\section{Extension to $H_{\infty}$ Control}

Consider the discrete-time switched system:

$$
\begin{aligned}
x(k+1) & =A_{i} x(k)+B_{i} u(k)+B_{w i} \omega(k), \\
z(k) & =C_{z i} x(k)+D_{u i} u(k), \\
y(k) & =C_{i} x(k),
\end{aligned}
$$

$$
\text { for } x \in S_{i}, i=1,2, \ldots, l \text {, }
$$

where $x(k) \in R^{n}, u(k) \in R^{m}, y(k) \in R^{p}, z(k) \in R^{q}$, and $\omega(k) \in$ $R^{r}$ are the state, the control input, the measured output, the control output, and the disturbance input, respectively; $S_{i} \subseteq$ $R^{n}, i=1,2, \ldots, l$, denotes a partition of the output space into a number of closed polyhedral regions. Let $\Omega$ be the set of all possible switches from one region to itself or another region; that is,

$$
\begin{aligned}
\Omega & =\left\{(i, j): i, j=1,2, \ldots, l \text { s.t. } x(k) \in S_{i}, x(k+1)\right. \\
& \left.\in S_{j}\right\} .
\end{aligned}
$$

With the controller (3), the closed-loop system of system (14) becomes

$$
\begin{aligned}
x(k+1) & =\left(A_{i}+B_{i} F_{i} C_{i}\right) x(k)+B_{w i} \omega(k), \\
z(k) & =\left(C_{z i}+D_{u i} F_{i} C_{i}\right) x(k), \quad i=1,2, \ldots, l .
\end{aligned}
$$

In this section, new sufficient conditions for SOF control design for the switched system (14) in the $H_{\infty}$ framework will be present. Given a scalar $\gamma>0$, assuming $x(0)=0$, the exogenous signal $\omega$ is attenuated by $\gamma$ if for each integer $N>0$ and for every $\omega \in L_{2}\left([0, N], R^{r}\right)$

$$
\sum_{k=0}^{N}\|z(k)\|^{2}<\gamma^{2} \sum_{k=0}^{N}\|\omega(k)\|^{2} .
$$

The $H_{\infty}$ performance of the closed-loop system (16) proposed by Cuzzola and Morari in [13] is reviewed in the next lemma.

Lemma 5 (see [13]). Consider the switched system (14), if there exists a function $V(x)=x^{T} P_{i} x, \forall x \in S_{i}$ with $P_{i}=P_{i}^{T}>0$ satisfying the following inequality:

$$
V(x(k+1))-V(x(k))<\gamma^{2}\|\omega(k)\|^{2}-\|z(k)\|^{2}, \quad, \quad \forall k ;
$$

then the closed-loop switched system (16) is exponentially stable with $H_{\infty}$ performance $\gamma$. 
Obviously, inequality (18) is equivalent to the following inequalities:

$$
\begin{aligned}
\left(A_{i}+\right. & \left.B_{i} F_{i} C_{i}\right)^{T} P_{j}\left(A_{i}+B_{i} F_{i} C_{i}\right) \\
& +\left(C_{z i}+D_{u i} F_{i} C_{i}\right)^{T}\left(C_{z i}+D_{u i} F_{i} C_{i}\right) \\
& +\left(A_{i}+B_{i} F_{i} C_{i}\right)^{T} P_{j} B_{w i} \Upsilon^{-1} B_{w i}^{T} P_{j}\left(A_{i}+B_{i} F_{i} C_{i}\right)
\end{aligned}
$$

$$
-P_{i}<0, \quad \forall(i, j) \in \Omega,
$$

where $\Upsilon=\gamma^{2} I-B_{w i}^{T} P_{j} B_{w i}>0$.

Now, the sufficient conditions to obtain SOF control gains with $H_{\infty}$ performance are given in the following theorem.

Theorem 6. If there exist points $\left(F_{i}(k), P_{i}(k)\right), i=1,2, \ldots, l$, such that the LMIs

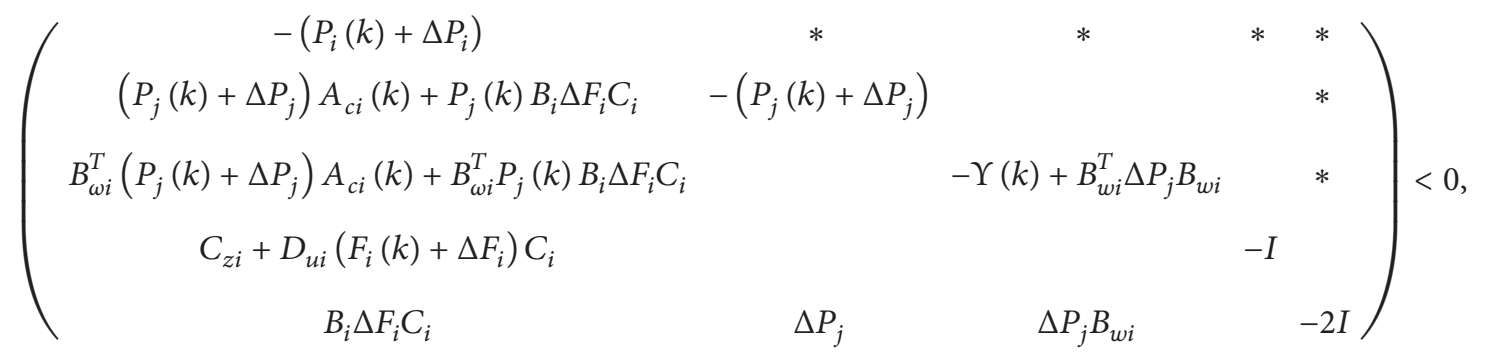

$\Upsilon(k)-B_{w i}^{T} \Delta P_{j} B_{w i}>0, P_{i}(k)+\Delta P_{i}>0, \forall(i, j) \in \Omega$

hold for some $\left(\Delta F_{i}, \Delta P_{i}\right)$, where $A_{c i}(k)=A_{i}+B_{i} F_{i}(k) C_{i}$ and $\Upsilon(k)=\gamma^{2} I-B_{w i}^{T} P_{j}(k) B_{w i}>0$, then the points $\left(P_{i}=\right.$ $\left.P_{i}(k)+\Delta P_{i}, F_{i}=F_{i}(k)+\Delta F_{i}\right)$ are feasible solutions to inequalities (19).

Proof. By Schur complement, inequalities (19) are equivalent to

$$
\left(\begin{array}{cccc}
-P_{i} & * & * & * \\
P_{j}\left(A_{i}+B_{i} F_{i} C_{i}\right) & -P_{j} & & \\
B_{w i}^{T} P_{j}\left(A_{i}+B_{i} F_{i} C_{i}\right) & & -\Upsilon & \\
C_{z i}+D_{u i} F_{i} C_{i} & & & -I
\end{array}\right)<0
$$

Write $F_{i}=F_{i}(k)+\Delta F_{i}, P_{i}=P_{i}(k)+\Delta P_{i}$, and $A_{c i}(k)=$ $A_{i}+B_{i} F_{i}(k) C_{i}$, where $F_{i}(k)$ and $P_{i}(k)$ are fixed matrices. The left side of inequality (21) is expanded around $\left(F_{i}(k), P_{i}(k)\right)$ as

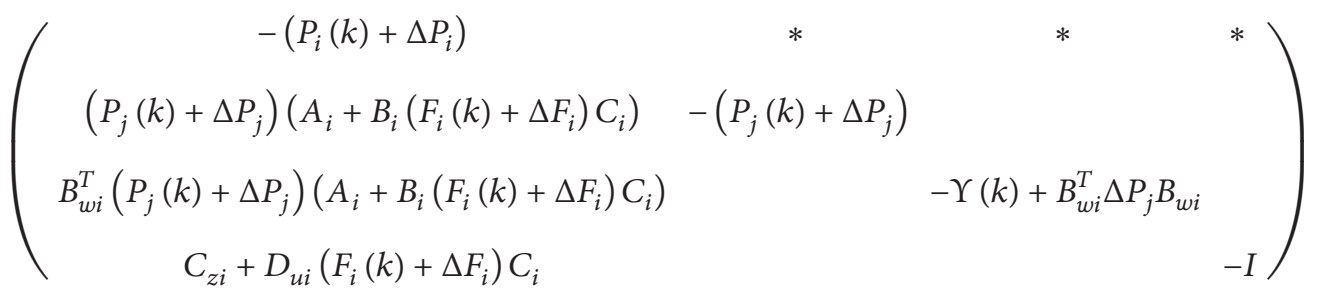

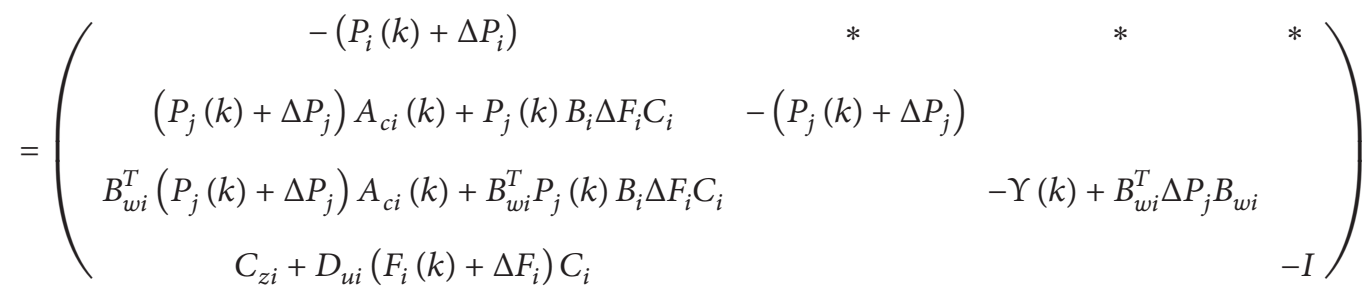

$$
\begin{aligned}
& +l\left(\left(B_{i} \Delta F_{i} C_{i} \quad 0 \quad 000\right)^{T},\left(\begin{array}{lllll}
0 & \Delta P_{j} & \Delta P_{j} B_{w i} & 0
\end{array}\right)\right) .
\end{aligned}
$$


That is, the following inequalities hold:

$$
\begin{aligned}
& l\left(\left(\begin{array}{llll}
B_{i} \Delta F_{i} C_{i} & 0 & 0 & 0
\end{array}\right)^{T},\left(\begin{array}{lllll}
0 & \Delta P_{j} & \Delta P_{j} B_{w i} & 0
\end{array}\right)\right)
\end{aligned}
$$

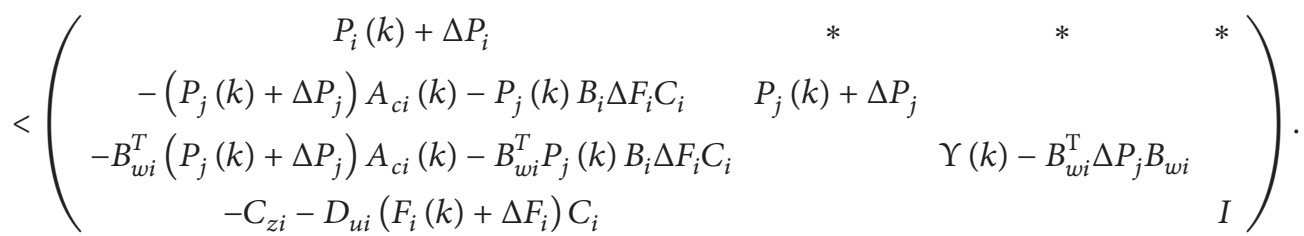

Thus, by Lemma 2, inequalities (20) hold.

With Theorem 6, the nonlinear optimization problem OP2 for solving SOF control with $H_{\infty}$ performance

$\min \alpha$

subject to $P_{i}>0$,

$$
\Upsilon=\gamma^{2} I-B_{w i}^{T} P_{j} B_{w i}>0
$$

$\min \alpha$

subject to $P_{i}(k)+\Delta P_{i}>0$,

$$
\begin{aligned}
& \Upsilon(k)-B_{w i}^{T} \Delta P_{j} B_{w i}>0, \\
& \left(\begin{array}{cc}
\beta P_{i}(k) & \Delta P_{i} \\
\Delta P_{i} & \beta P_{i}(k)
\end{array}\right)>0,
\end{aligned}
$$$$
\left(\begin{array}{c}
-\left(P_{i}(k)+\Delta P_{i}\right)-\alpha P_{i}(k) \\
\left(P_{j}(k)+\Delta P_{j}\right) A_{c i}(k)+P_{j}(k) B_{i} \Delta F_{i} C_{i} \\
B_{\omega i}^{T}\left(P_{j}(k)+\Delta P_{j}\right) A_{c i}(k)+B_{\omega i}^{T} P_{j}(k) B_{i} \Delta F_{i} C_{i} \\
C_{z i}+D_{u i}\left(F_{i}(k)+\Delta F_{i}\right) C_{i} \\
B_{i} \Delta F_{i} C_{i}
\end{array}\right.
$$

$<0$,

Based on Theorems 3 and 6, an iterative algorithm to solve stabilization and $H_{\infty}$ control via static output feedback for discrete-time switched systems is established.

Algorithm 7. Consider the following.

Step 1 (initialization step). At initial, we need to obtain initial values of $F_{i}$ and $P_{i}$.

$$
\left(\begin{array}{cccc}
-P_{i}-\alpha P_{i} & * & * & * \\
P_{j}\left(A_{i}+B_{i} F_{i} C_{i}\right) & -P_{j} & & \\
B_{w i}^{T} P_{j}\left(A_{i}+B_{i} F_{i} C_{i}\right) & & -\Upsilon & \\
C_{z i}+D_{u i} F_{i} C_{i} & & & -I
\end{array}\right)
$$$$
<0
$$$$
\forall(i, j) \in \Omega
$$

can be replaced by solving the following linear optimization problem OP2': 
$\mathrm{OP}^{\prime}$ (or OP2 ${ }^{\prime}$ instead for $H_{\infty}$ case) with respect to $\Delta F_{i}, \Delta P_{i}$, and $\alpha$.

Step 3 (update step). Let $F_{i}(k)=F_{i}(k-1)+\Delta F_{i}, P_{i}(k)=P_{i}(k-$ $1)+\Delta P_{i}, \alpha(k)=\alpha$, and $A_{c i}(k)=A_{c i}(k-1)+B_{i} \Delta F_{i} C_{i}$. For fixed $F_{i}(k)$, compute new $P_{i}(k)$ by solving OP1 (or OP2 instead for $H_{\infty}$ case), and then compute new $F_{i}(k)$ and $\alpha(k)$ by solving OP1 (or OP2 instead for $H_{\infty}$ case).

If $\alpha(k)<0$, stop; else if the relative improvement in $\alpha$ is more than a desired accuracy, set $k=k+1$, and go to Step 2 . Else, set $k=1$, and let $F_{i}(0)=F_{i}(k), P_{i}(0)=P_{i}(k), A_{c i}(0)=$ $A_{c i}(k)$, and $\alpha(0)=\alpha(k)$.

Step 4 (wide perturbation step). Set $\beta=\beta \times 2$. Solve LMI optimization problem OP1' (or OP2' instead for $H_{\infty}$ case) with respect to $\Delta F_{i}, \Delta P_{i}$, and $\alpha$.

Step 5 (update step). Let $F_{i}(k)=F_{i}(k-1)+\Delta F_{i}, P_{i}(k)=P_{i}(k-$ $1)+\Delta P_{i}, \alpha(k)=\alpha$, and $A_{c i}(k)=A_{c i}(k-1)+B_{i} \Delta F_{i} C_{i}$. For fixed $F_{i}(k)$, compute new $P_{i}(k)$ by solving OP1 (or OP2 instead for $H_{\infty}$ case), and then compute new $F_{i}(k)$ and $\alpha(k)$ by solving OP1 (or OP2 instead for $H_{\infty}$ case).

If $\alpha(k)<0$, stop; else if the relative improvement in $\alpha$ is more than a desired accuracy, set $k=1$, and let $F_{i}(0)=F_{i}(k)$, $P_{i}(0)=P_{i}(k), A_{c i}(0)=A_{c i}(k)$, and $\alpha(0)=\alpha(k)$, and go to Step 2. Else if the relative improvement in $\alpha$ is inferior to the desired accuracy and $k<k_{0}$, where $k_{0}>0$ is a prescribed integer, set $k=k+1$, and go to Step 4. Else, stop.

Remark 8. The wide perturbation step is a crucial step in improved path-following method. The purpose of this step is to broaden the search scope during each iteration so that the algorithm has the opportunity to escape form the local optimum. However, the enlarged search scope may cause nonconvergence. So the iteration number of wide perturbation step should not be too large. As long as the objective function $\alpha$ has been improved significantly, the wide perturbation step will be replaced by a small perturbation step immediately.

\section{Numerical Examples}

In this section, two examples are given to show the effectiveness of our method. Example 1 is with respect to the SOF control problem for switched systems. Example 2 is concerning the $H_{\infty}$ controller design problem for switched systems.

Example 1. Consider system (1) with the following parameters:

$$
\begin{aligned}
& A_{1}=\left(\begin{array}{ccc}
1 & 0.3 & 2 \\
1 & 0 & 1 \\
0.3 & 0.6 & 0.6
\end{array}\right), \\
& A_{2}=\left(\begin{array}{ccc}
-0.5871 & -0.8441 & -0.0092 \\
-0.6865 & -0.5090 & -0.8561 \\
0.0974 & 0.4523 & -0.2280
\end{array}\right),
\end{aligned}
$$

$$
\begin{aligned}
& A_{3}=\left(\begin{array}{ccc}
0.1089 & 0.2458 & -0.9035 \\
0.3998 & -0.9213 & -0.4161 \\
0.6745 & -0.5750 & 0.7138
\end{array}\right) \text {, } \\
& B_{1}=\left(\begin{array}{ll}
1 & 0 \\
0 & 1 \\
1 & 0
\end{array}\right), \\
& B_{2}=\left(\begin{array}{cc}
0.1930 & -0.4204 \\
-0.7359 & 0.0346 \\
0.5073 & -0.9077
\end{array}\right) \text {, } \\
& B_{3}=\left(\begin{array}{cc}
-0.4164 & 0.0244 \\
0.8297 & -0.4366 \\
-0.0900 & -0.8416
\end{array}\right) \text {, } \\
& C_{1}=\left(\begin{array}{lll}
1 & 1 & 0
\end{array}\right), \\
& C_{2}=\left(\begin{array}{lll}
1 & 0 & 1
\end{array}\right), \\
& C_{3}=\left(\begin{array}{lll}
0 & 1 & 1
\end{array}\right) .
\end{aligned}
$$

It cannot be stabilized by the method in $[12,20]$. However, using our method, set $\beta_{0}=0.2$; after iterations, output feedback stabilizing controller matrices are computed to be

$$
\begin{aligned}
& F_{1}=\left(\begin{array}{l}
-0.7454 \\
-0.8806
\end{array}\right), \\
& F_{2}=\left(\begin{array}{c}
-0.8899 \\
-0.2598
\end{array}\right), \\
& F_{3}=\left(\begin{array}{lll}
0.5736 \\
0.4941
\end{array}\right), \\
& P_{1}=\left(\begin{array}{ccc}
11359.9012 & -13835.4590 & 3078.1446 \\
-13835.4590 & 45253.4272 & -20668.2151 \\
3078.1446 & -20668.2151 & 36198.7545
\end{array}\right), \\
& P_{2}=\left(\begin{array}{ccc}
7437.9005 & -6354.0674 & -141.5298 \\
-6354.0674 & 34828.7955 & -8170.2774 \\
-141.5298 & -8170.2774 & 16785.3283
\end{array}\right), \\
& P_{3}=\left(\begin{array}{ccc}
12867.1197 & -18832.8739 & 5382.2570 \\
-18832.8739 & 43961.3298 & -14881.7520 \\
5382.2570 & -14881.7520 & 23348.2946
\end{array}\right) .
\end{aligned}
$$


Example 2. Consider a system with the following parameters:

$$
\begin{aligned}
& A_{1}=\left(\begin{array}{ccc}
-0.5871 & -0.8441 & -0.0092 \\
-0.6865 & -0.5090 & -0.8561 \\
0.0974 & 0.4523 & -0.2280
\end{array}\right) \text {, } \\
& A_{2}=\left(\begin{array}{ccc}
0.1089 & 0.2458 & -0.9035 \\
0.3998 & -0.9213 & -0.4161 \\
0.6745 & -0.5750 & 0.7138
\end{array}\right) \text {, } \\
& B_{1}=\left(\begin{array}{cc}
0.1930 & -0.4204 \\
-0.7359 & 0.0346 \\
0.5073 & -0.9077
\end{array}\right) \text {, } \\
& B_{2}=\left(\begin{array}{cc}
-0.4164 & 0.0244 \\
0.8297 & -0.4366 \\
-0.0900 & -0.8416
\end{array}\right) \text {, } \\
& C_{1}=\left(\begin{array}{lll}
1 & 0 & 1
\end{array}\right), \\
& C_{2}=\left(\begin{array}{lll}
0 & 1 & 1
\end{array}\right), \\
& B_{w 1}=B_{w 2}=\left(\begin{array}{l}
0 \\
1 \\
0
\end{array}\right) \\
& C_{z 1}=C_{z 2}=\left(\begin{array}{lll}
1 & 0 & 0 \\
0 & 1 & 0 \\
0 & 0 & 1
\end{array}\right) \text {, } \\
& D_{u 1}=D_{u 2}=\left(\begin{array}{ll}
0 & 0 \\
0 & 1 \\
0 & 1
\end{array}\right)
\end{aligned}
$$

Using our method, set $\beta_{0}=0.2$; after iterations, output feedback control matrices are computed to be

$$
\begin{aligned}
& F_{1}=\left(\begin{array}{c}
-0.9811 \\
-0.6662
\end{array}\right), \\
& F_{2}=\left(\begin{array}{c}
0.4284 \\
-0.2097
\end{array}\right) .
\end{aligned}
$$

In this case,

$$
\begin{aligned}
& P_{1}=\left(\begin{array}{ccc}
5.3254 & -2.9578 & 1.2843 \\
-2.9578 & 12.6330 & -2.3378 \\
1.2843 & -2.3378 & 2.8444
\end{array}\right), \\
& P_{2}=\left(\begin{array}{ccc}
6.0132 & -3.0439 & 3.3919 \\
-3.0439 & 6.3742 & -3.3469 \\
3.3919 & -3.3469 & 9.2144
\end{array}\right)
\end{aligned}
$$

and the closed-loop system has the $H_{\infty}$ performance $\gamma=$ 4.7328. The result is better than the solution solved by the method in [12] which gives $\gamma=5.6853$, which is inferior compared to our result $\gamma=4.7328$ and

$$
\begin{aligned}
& F_{1}=\left(\begin{array}{c}
-1.0832 \\
-0.5259
\end{array}\right), \\
& F_{2}=\left(\begin{array}{c}
0.3563 \\
-0.1241
\end{array}\right) .
\end{aligned}
$$

\section{Conclusion}

This paper studies the problems of static output feedback control and $H_{\infty}$ controller synthesis for discrete-time switched systems. Based on piecewise quadratic Lyapunov functions and a new linearization method, new sufficient conditions for system stability and $H_{\infty}$ controller design are obtained. Then, an improved path-following algorithm is built to solve the problems. Finally, the merits and effectiveness of the proposed method are shown by two numerical examples. Compared to the existing methods, the proposed method is less conservative.

Important future research work will be applying the results to some real-world systems. How to reduce the design conservatism is an important research topic that deserves further investigation.

\section{Conflict of Interests}

The authors declare that there is no conflict of interests regarding the publication of this paper.

\section{Acknowledgments}

This work was supported in part by the National Natural Science Foundation of China (nos. 61174033 and 61473160) and in part by the Natural Science Foundation of Shandong Province, China (ZR2011FM006).

\section{References}

[1] J. Xu and L. Xie, "Null controllability of discrete-time planar bimodal piecewise linear systems," International Journal of Control, vol. 78, no. 18, pp. 1486-1496, 2005.

[2] L. Rodrigues and J. P. How, "Automated control design for a piecewise-affine approximation of a class of nonlinear systems," in Proceedings of the American Control Conference, pp. 31893194, Arlington, Va, USA, June 2001.

[3] Z. Sun and S. S. Ge, "Analysis and synthesis of switched linear control systems," Automatica, vol. 41, no. 2, pp. 181-195, 2005.

[4] L. Rodrigues and J. P. How, "Observer-based control of piecewise-affine systems," International Journal of Control, vol. 76, no. 5, pp. 459-477, 2003.

[5] V. D. Blondel and J. N. Tsitsiklis, "Complexity of stability and controllability of elementary hybrid systems," Automatica, vol. 35, no. 3, pp. 479-489, 1999.

[6] Z. Ji, L. Wang, and G. Xie, "Quadratic stabilization of uncertain discrete-time switched systems via output feedback," Circuits, Systems, and Signal Processing, vol. 24, no. 6, pp. 733-751, 2005. 
[7] H. Lin and P. J. Antsaklis, "Hybrid state feedback stabilization with $l_{2}$ performance for disrete-time switched linear systems," International Journal of Control, vol. 81, no. 7, pp. 1114-1124, 2008.

[8] G. S. Deaecto, J. C. Geromel, and J. Daafouz, "Dynamic output feedback $H_{\infty}$ control of switched linear systems," Automatica, vol. 47, pp. 1713-1720, 2011.

[9] J. C. Geromel, P. Colaneri, and P. Bolzern, "Dynamic output feedback control of switched linear systems," IEEE Transactions on Automatic Control, vol. 53, no. 3, pp. 720-733, 2008.

[10] G.-H. Yang and J. X. Dong, "Switching fuzzy dynamic output feedback $H_{\infty}$ control for nonlinear systems," IEEE Transactions on Systems, Man, and Cybernetics, Part B: Cybernetics, vol. 40, no. 2, pp. 505-516, 2010.

[11] J. X. Dong and G.-H. Yang, " $H_{\infty}$ controller synthesis via switched PDC scheme for discrete-time T-S fuzzy systems," IEEE Transactions on Fuzzy Systems, vol. 17, no. 3, pp. 544-555, 2009.

[12] D.-W. Ding and G.-H. Yang, "Static output feedback control for discrete-time piecewise linear systems: an LMI approach," Acta Automatica Sinica, vol. 35, no. 4, pp. 337-344, 2009.

[13] F. A. Cuzzola and M. Morari, "An LMI approach for $H_{\infty}$ analysis and control of discretetime piecewise affine systems," International Journal of Control, vol. 75, no. 16-17, pp. 1293-1301, 2002.

[14] K. C. Goh, L. Turan, M. G. Safonov, G. P. Papavassilopoulos, and J. H. Ly, "Biaffine matrix inequality properties and computational methods," in Proceedings of the American Control Conference, vol. 1, pp. 850-855, IEEE, June-July 1994.

[15] A. Hassibi, J. P. How, and S. P. Boyd, "Path-following method for solving BMI problems in control," in Proceedings of the American Control Conference, pp. 1385-1389, Evanston, Ill, USA, June 1999.

[16] E. Ostertag, "An improved path-following method for mixed $\mathrm{H}_{2} / \mathrm{H}_{\infty}$ controller design," IEEE Transactions on Automatic Control, vol. 53, no. 8, pp. 1967-1971, 2008.

[17] E. Ostertag, "Continuous- and discrete- time path-following design of mixed $\mathrm{H}_{2} / \mathrm{H}_{\infty}$ state-feedback controllers," in Proceedings of the 17th World Congress: The International Federation of Automatic Control, pp. 3988-3993, Seoul, Republic of Korea, July 2008.

[18] M. I. Syam, H. I. Siyyam, and I. Al-Subaihi, “Tau-path following method for solving the riccati equation with fractional order," Journal of Computational Methods in Physics, vol. 2014, Article ID 207916, 7 pages, 2014.

[19] J. Chen and C. Lin, "BMI optimization based on improved pathfollowing method in control," in Proceedings of the 2015 Chinese Intelligent Automation Conference, vol. 337 of Lecture Notes in Electrical Engineering, pp. 127-134, Springer, Berlin, Germany, 2015.

[20] G. I. Bara and M. Boutayeb, "Switched output feedback stabilization of discrete-time switched systems," in Proceedings of the 45th IEEE Conference on Decision and Control, pp. 2667-2672, San Diego, Calif, USA, December 2006. 


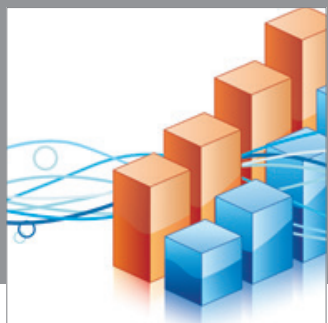

Advances in

Operations Research

mansans

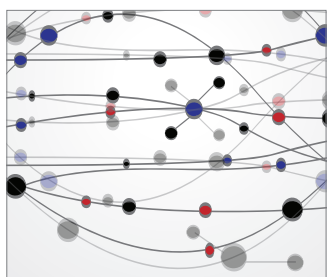

The Scientific World Journal
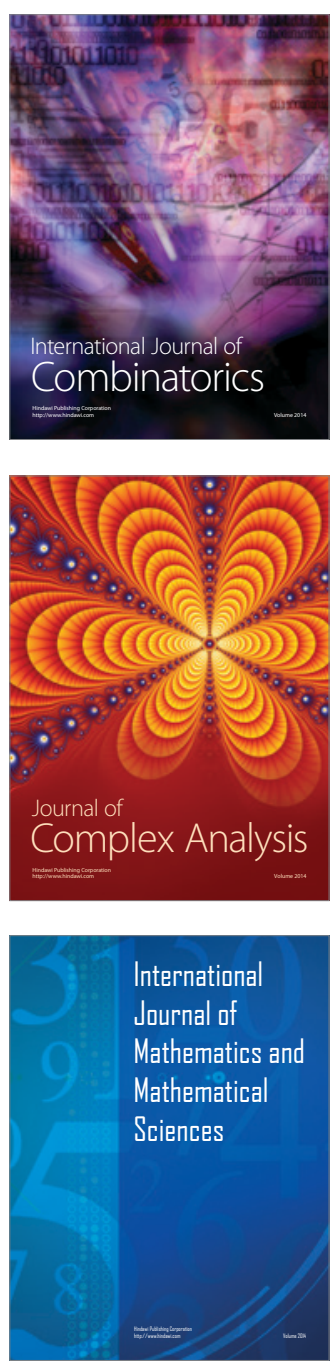
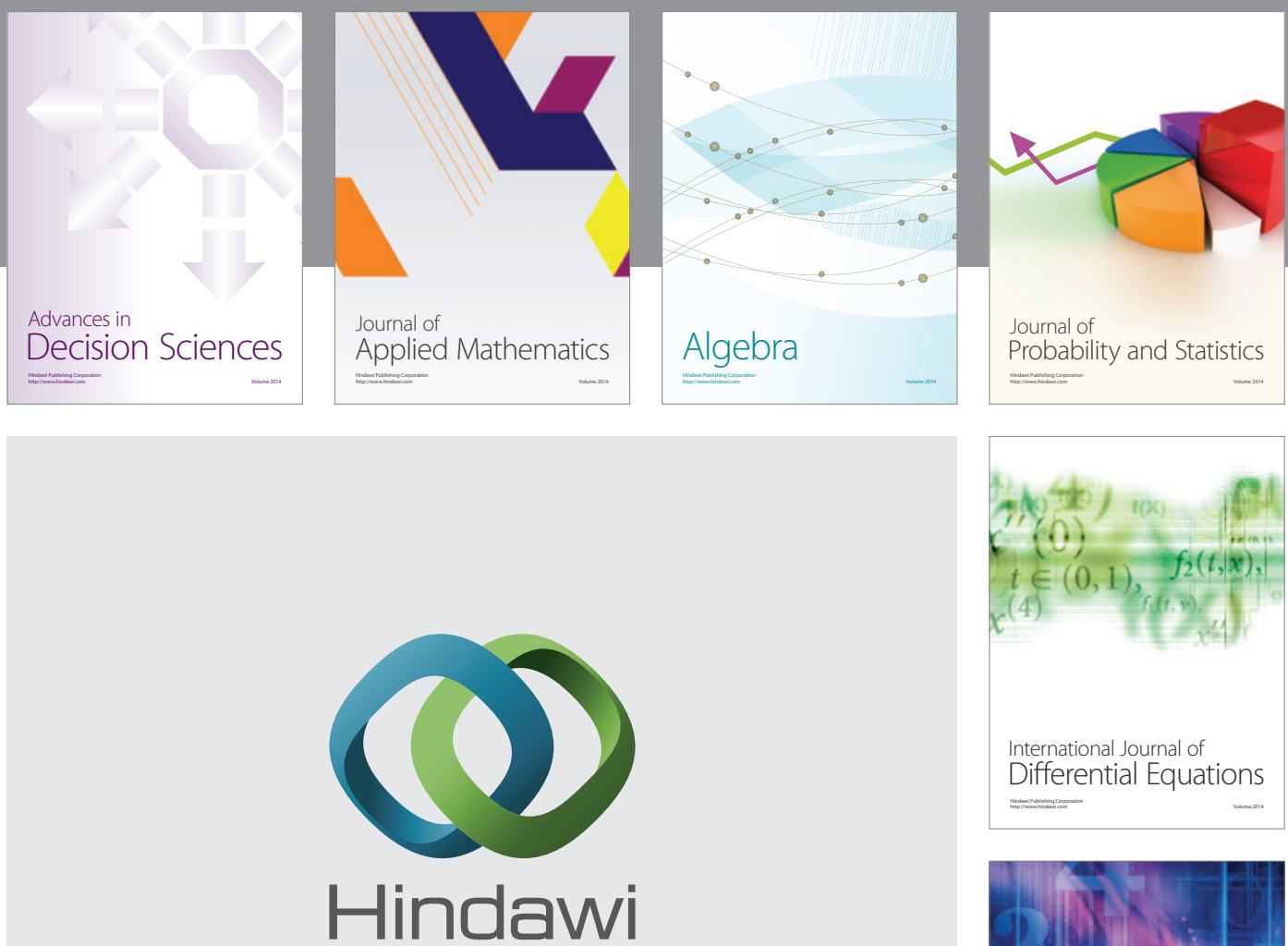

Submit your manuscripts at http://www.hindawi.com
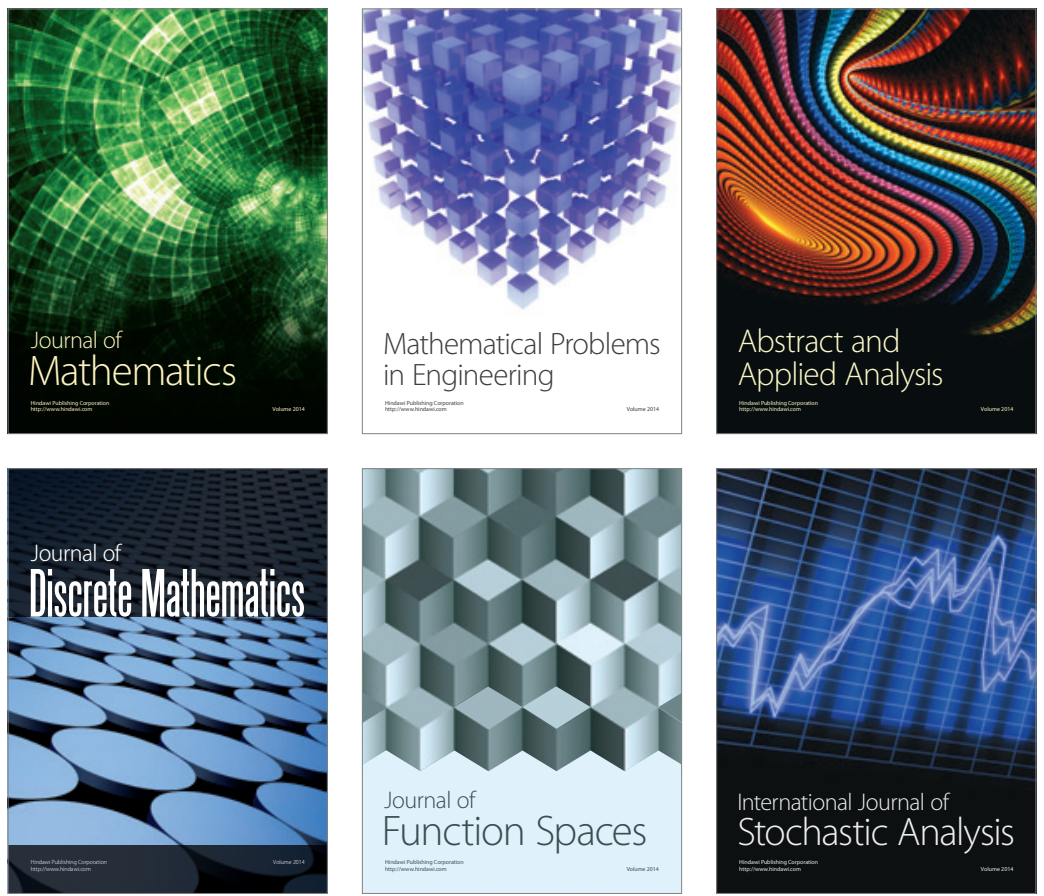

Journal of

Function Spaces

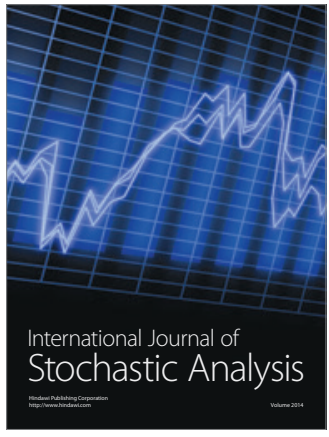

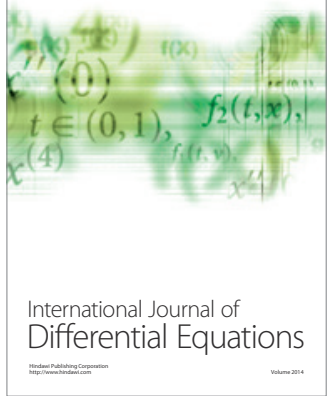
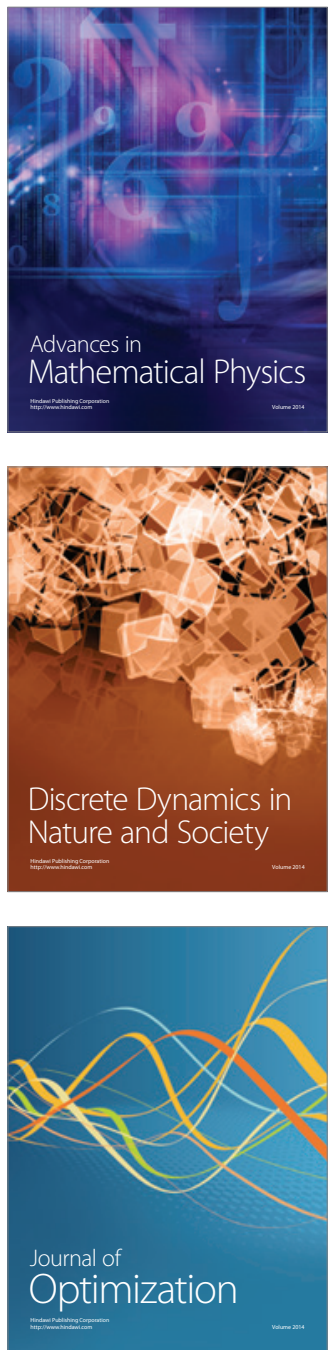